\title{
Instruments and Methods Density measurements in ice boreholes using neutron scattering
}

\author{
Elizabeth M. MORRIS, ${ }^{*}{ }^{*}$ J. David COOPER ${ }^{2}$ \\ ${ }^{1}$ British Antarctic Survey, Natural Environment Research Council, Madingley Road, Cambridge CB3 OET, England \\ E-mail:emmo@nerc-bas.ac.uk \\ ${ }^{2}$ CEH-Wallingford, Maclean Building, Crowmarsh Gifford, Wallingford, Oxfordshire OX10 8BB, England
}

\begin{abstract}
This paper describes the use of a neutron probe to measure detailed stratigraphy in ice and snow. The Wallingford neutron probe, developed for measurement of soil moisture, consists of an annular radioactive source of fast neutrons around the centre of a cylindrical detector for slow (thermal) neutrons. In snow and ice, the fast neutrons lose energy by scattering from hydrogen atoms, and the number of slow neutrons arriving at the detector (the count rate) is related to the density of the medium. Calibration equations for count rate as a function of snow density and borehole diameter have been derived. Snow-density profiles from boreholes obtained using the probe show that, despite the smoothing produced by the neutron-scattering process, annual variations in density can be resolved. The potential contribution of the neutron probe to improvements in mass-balance monitoring is discussed.
\end{abstract}

\section{INTRODUCTION}

Ice caps in the circum-arctic area undergo a varying degree of surface summer melting. Study of ice layering in ice cores from these ice caps provides a detailed picture of summer climate changes to complement the traditional stable-isotope approach (e.g. Koerner, 1997). These clear layers of density $900-914 \mathrm{~kg} \mathrm{~m}^{-3}$ can be resolved against the background seasonal variations in ice-core density (which decrease from up to $\pm 25 \%$ at the surface to $\pm 1-5 \%$ at depth) by visual inspection or automatic laser profiling of the ice-core segments (e.g. Zagarodnov and others, 1994). The overall depth-density function also contains key information on accumulation and surface temperature conditions. It can be measured by weighing or automatic gamma-ray profiling of ice-core segments (e.g. Gerland and others, 1999). The disadvantage of these methods is that the data are discontinuous at core breaks. This paper describes an alternative, down-borehole, method of density measurement using neutron scattering.

\section{THE WALLINGFORD PROBE}

The Wallingford probe was developed for measurement of soil moisture profiles using vertical access holes augered in the soil (Bell, 1969). The probe consists of an annular radioactive source of fast neutrons around the centre of a cylindrical detector for slow (thermal) neutrons. The fast

* Present address: Scott Polar Research Institute, University of Cambridge, Lensfield Road, Cambridge CB2 1ER, England. neutrons lose energy by scattering, especially from the light hydrogen atoms in soil water, and the number of slow neutrons arriving at the detector per unit time is related to the soil moisture content. In the case of water, snow or ice, the neutron current at the detector is related to the density of the medium. Note that, by convention, the flux of neutrons in a given direction is known as the "neutron current". The term "neutron flux" is used for a scalar variable: the neutron track length per unit time per unit volume.

The source/detector assembly is lowered into the access hole on a cable. The electrical pulses produced by the detector as each thermal neutron is absorbed are amplified and shaped before passing up the cable to a rate scaler where the mean count rate over a set period is displayed as counts per second. Counting periods of $16 \mathrm{~s}, 64 \mathrm{~s}, 16 \mathrm{~min}$ and $64 \mathrm{~min}$ are available. The source emits neutrons randomly, so for $N$ counts taken over time $t$ the count rate, $R$, and standard error, $\sigma_{R}$, are given by

$$
R \pm \sigma_{R}=\frac{N}{t} \pm \frac{\sqrt{N}}{t}
$$

Thus, longer counting times give more precise count rates.

The source is an annular americium-241/beryllium neutron source of nominal strength $1.85 \mathrm{GBq}$. It is encapsulated in a stainless-steel AEA Technology X.20 capsule of length $19 \mathrm{~mm}$, internal diameter $26.2 \mathrm{~mm}$ and external diameter $33.3 \mathrm{~mm}$. The tolerance on source strength is $10 \%$, so that the expected neutron emission is $q_{0}=(1.1 \pm$ $0.1) \times 10^{5}$ neutron $\mathrm{s}^{-1}$. The neutron spectrum is shown in Figure 1. The average energy of the neutrons above $2 \mathrm{MeV}$ is $\approx 4.5 \mathrm{MeV}$. Note, however, that the source also emits a significant number of lower-energy neutrons $(\approx 23 \%$ below $1 \mathrm{MeV}$ with mean energy $400 \mathrm{keV}$ ).

The source is fitted around the centre of an enriched 


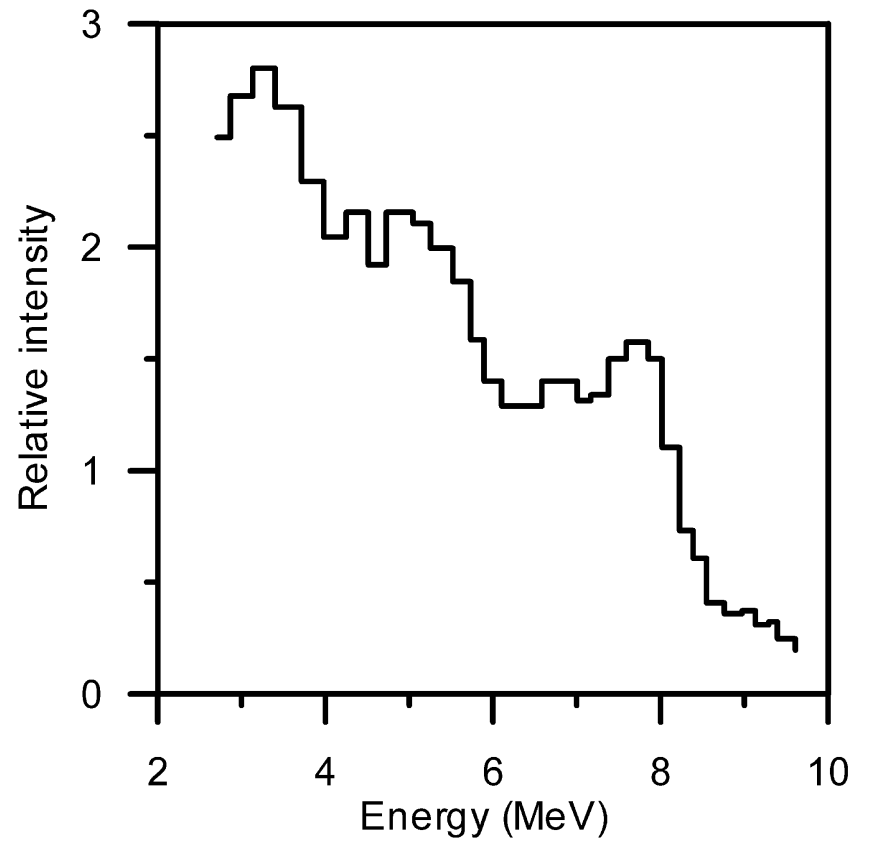

Fig. 1. The neutron spectrum of the Wallingford probe source (from AEA Technology data sheet). The relative number of neutrons for a given energy interval (relative intensity) is given on a linear scale.

boron trifluoride proportional counter (Centronic Ltd UK type $12 \mathrm{~EB} 70 \mathrm{G}$ ). The nominal count rate per unit neutron flux through the detector is $5.2 \mathrm{cps} /$ neutron $\mathrm{cm}^{-2} \mathrm{~s}^{-1}$. The counter has an active length of $135 \mathrm{~mm}$, nominal outside diameter 1 in $(25.4 \mathrm{~mm})$ and internal diameter $24.4 \mathrm{~mm}$. The active volume is therefore $63.02 \mathrm{~cm}^{3}$, implying a nominal macroscopic absorption cross-section of $8.251 \mathrm{~m}^{-1}$.

\section{EXPERIMENTAL GALIBRATION}

Calibration of a neutron probe for snow-density measurement is based on measurement of a "water count" $R_{\mathrm{W} D}$ which is the thermal neutron count rate when the probe is in an access tube of diameter $D$ surrounded by water of density $\rho_{\mathrm{w}}$. Kane (1969) suggested that the calibration equation relating count rate $R_{D}$ to snow density $\rho_{\mathrm{s}}$ should have the form

$$
R_{D}=R_{\mathrm{W} D}\left[\rho_{\mathrm{s}} / \rho_{\mathrm{w}}\right]^{x}
$$

and that, for finite detector size and access tube diameter, the value of $x$ should lie in the range $1-1.5$. This statement was based on an error in scaling in his Fermi age theory for neutron transport in snow (Fermi age theory in fact leads to $x=3$ ), but nevertheless empirical calibration data for the Nuclear Chicago DM-19 probe (Anderson and others, 1962; Leighty, 1965) do fit the equation with $x=1.28$.

Figure 2 shows empirical calibration data for the Wallingford probe obtained by comparing count rate in a $9 \mathrm{~cm}$ diameter borehole (D98) on Devon Ice Cap, Nunavut, Canada, with the densities of core segments of around $10 \mathrm{~cm}$ length taken from corresponding depths. The neutron-probe data are representative of a sphere of about this radius around the source/detector unit, although the snow closest to the unit has the most effect on the count rate. The probe was not fitted with a centring device. The snow densities range from 400 to $900 \mathrm{~kg} \mathrm{~m}^{-3}$ and have been divided by the density of water. The data fit the power-law calibration

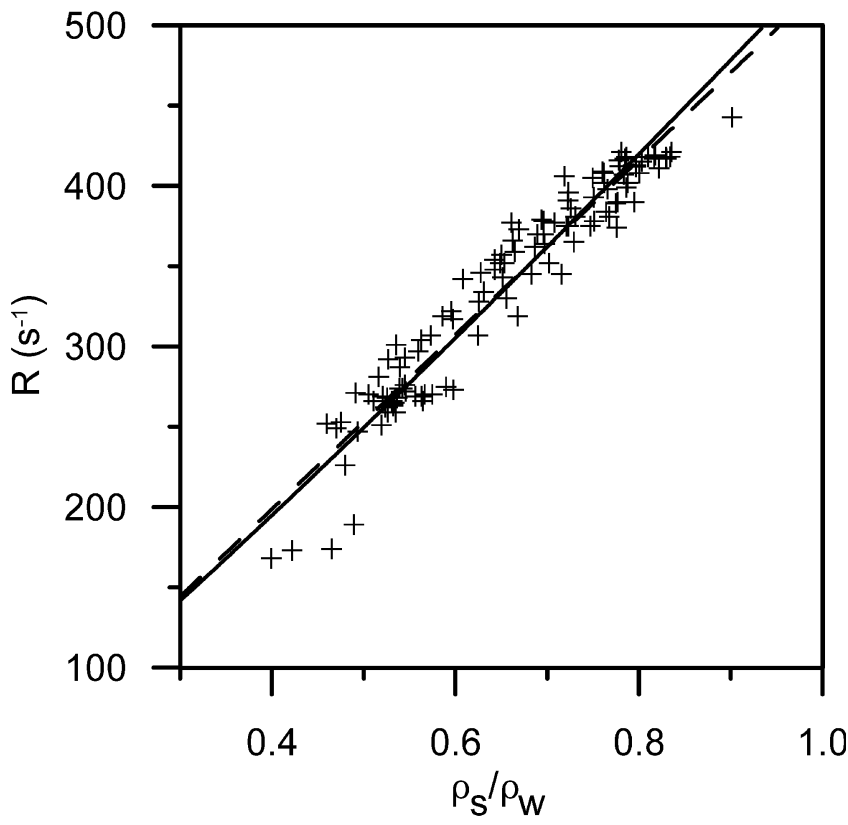

Fig. 2. Calibration curves for the Wallingford neutron probe relating neutron count rate per second to the relative density of snow: (a) power-law equation (solid line); ( b ) linear equation (dashed line).

equation (Equation (2)) with $x=1.11 \pm 0.03$ and $r^{2}=0.91$. However, they also fit a linear equation

$$
\left(1-\rho_{\mathrm{s}} / \rho_{\mathrm{W}}\right)=A_{D}\left(1-R_{D} / R_{\mathrm{W} D}\right)
$$

equally well with $r^{2}=0.93$. The scatter of \pm 0.05 in the relative density at a given count rate is not unexpected for gravimetric measurements of core segment density (e.g. Anderson and others (1962) quote a mean error in relative snow density of \pm 0.036 for their calibration data) but means that this type of field data cannot be used to determine the best form of the calibration equation, only the best parameters, once the form has been chosen.

One method of choosing the appropriate form is by theoretical analysis of the physics of the process. Olgaard (1965) has shown that, for neutron scattering from a point source in water, good approximate solutions can be obtained by grouping the neutrons into three energy ranges with three constant slowing-down lengths, $L, L_{1}, L_{2}$. The fast, medium and slow neutron fluxes are calculated using diffusion theory, with the transfer of neutrons from the fast to medium and then to the slow group determined by the scattering cross-section for each group. For a Ra-Be source which, like the Wallingford source, emits neutrons with average energy of about $4.5 \mathrm{MeV}$, Ølgaard grouped neutrons with energies of 4.5-2 MeV in the "fast" group, $2 \mathrm{MeV}$ to $5 k T_{\mathrm{n}}$ in the "medium" group and $<5 k T_{\mathrm{n}}$ in the slow group. $T_{\mathrm{n}}$ is the neutron temperature and $k$ is Boltzmann's constant.

For snow, the diffusion coefficients and slowing-down lengths increase with decreasing density (e.g. $L_{\mathrm{s}}=L_{\mathrm{w}} \rho_{\mathrm{w}} / \rho_{\mathrm{s}}$. That is, the lengths defined by the physics of the problem all scale with relative density. For a point source and point sink of neutrons co-located on the axis of the access hole, there is only one length scale defined by the geometry of the problem: the diameter of the access hole, $D$. Thus the count rate should be a function of only one variable, the ratio of the scales, $\rho_{\mathrm{s}} / D \rho_{\mathrm{w}}$. This relation may break down as $D$ approaches the length scale of the detector, which then must be regarded as having finite size, but, even 


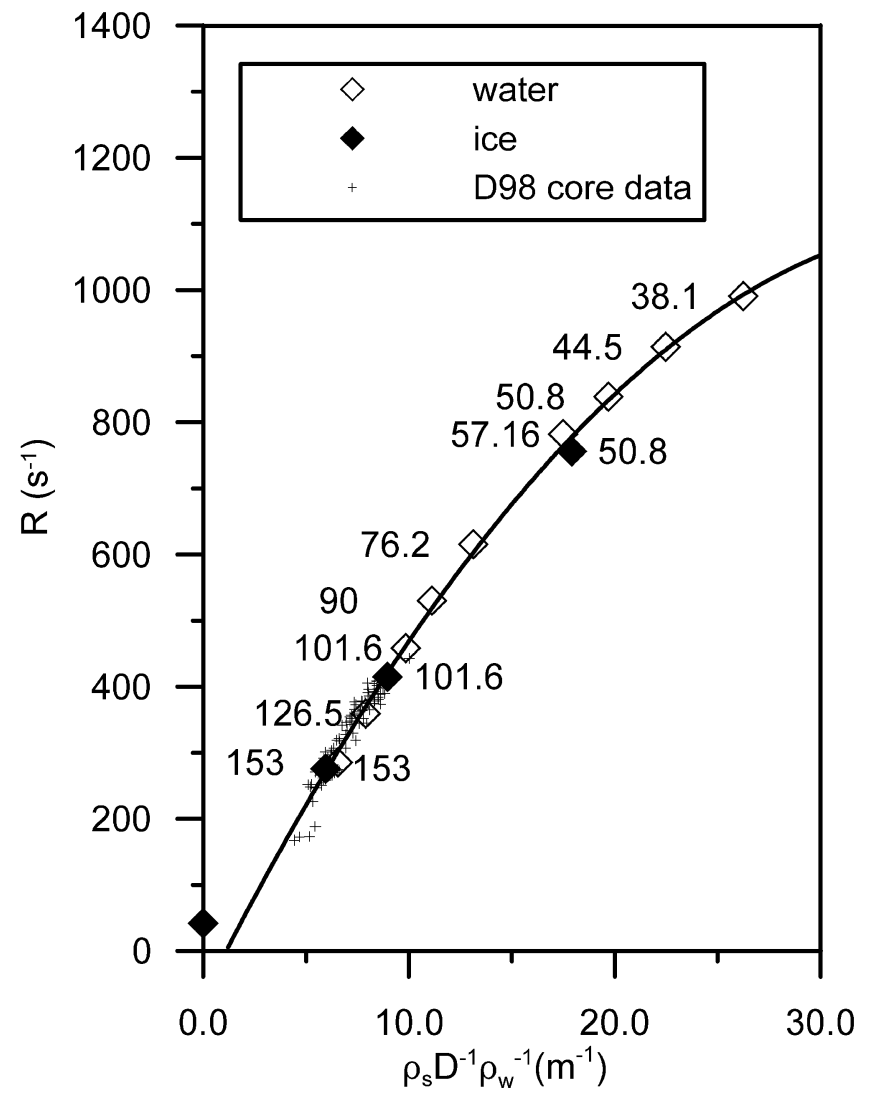

Fig. 3. Calibration curve for the Wallingford neutron probe relating neutron count rate per second to the relative density of snow and the diameter of the access tube. The numbers on the plot are the diameters of the access tubes in millimetres.

so, it is clearly possible to arrive at an estimate of the variation of count rate with snow density for constant diameter by measuring the variation of count rate with diameter at constant density. This is what we have done.

Using the methods laid down by the Institute of Hydrology (1981), we have determined the variation of $R_{\mathrm{W} D}$ with $D$ by measuring the standard count rate over $16 \mathrm{~min}$ in a series of five $3.25 \mathrm{~mm}$ thick aluminium access tubes of outside

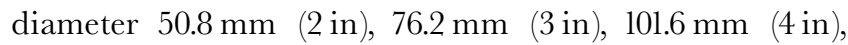
$126.54 \mathrm{~mm}$ ( $5 \mathrm{in}$ ) and $152.96 \mathrm{~mm}$ (6 in) surrounded by water in a large test tank. The tubes were $0.9 \mathrm{~m}$ long and sealed by a flat plate at the lower end attached to a weight to offset the upthrust of the surrounding medium. Further values of $R_{\text {WD }}$ have been deduced from fitting the linear calibration equation to field data from boreholes with nominal diameters of 57.15 and $90 \mathrm{~mm}$. We have also measured the count rate in solid ice, $R_{\mathrm{ID}}$, using three of the access tubes. Ice counts were obtained by freezing water around the access tubes as slowly as possible in an attempt to reject dissolved air at the freezing fronts. This was not entirely successful and the ice was not bubble-free. Thus the density of the medium was slightly less than that of pure ice.

The experimental count rates are shown in Figure 3 plotted against the scale ratio $\rho_{\mathrm{s}} / D \rho_{\mathrm{w}}$. We have shown the count rate in air $(42 \mathrm{cps})$ at the origin of the scale ratio axis, i.e. when $\rho_{\mathrm{s}} \rightarrow 0$ and/or $D \rightarrow \infty$. The ice and water data lie on the same curve, confirming that the simple scaling approach may be used to deduce the appropriate form of the calibration equation. A quadratic calibration equation with coefficients $a_{0}=-67.7, a_{1}=61.8, a_{2}=-0.816$ and $r^{2}=0.98$ is shown. The snow data shown in Figure 2

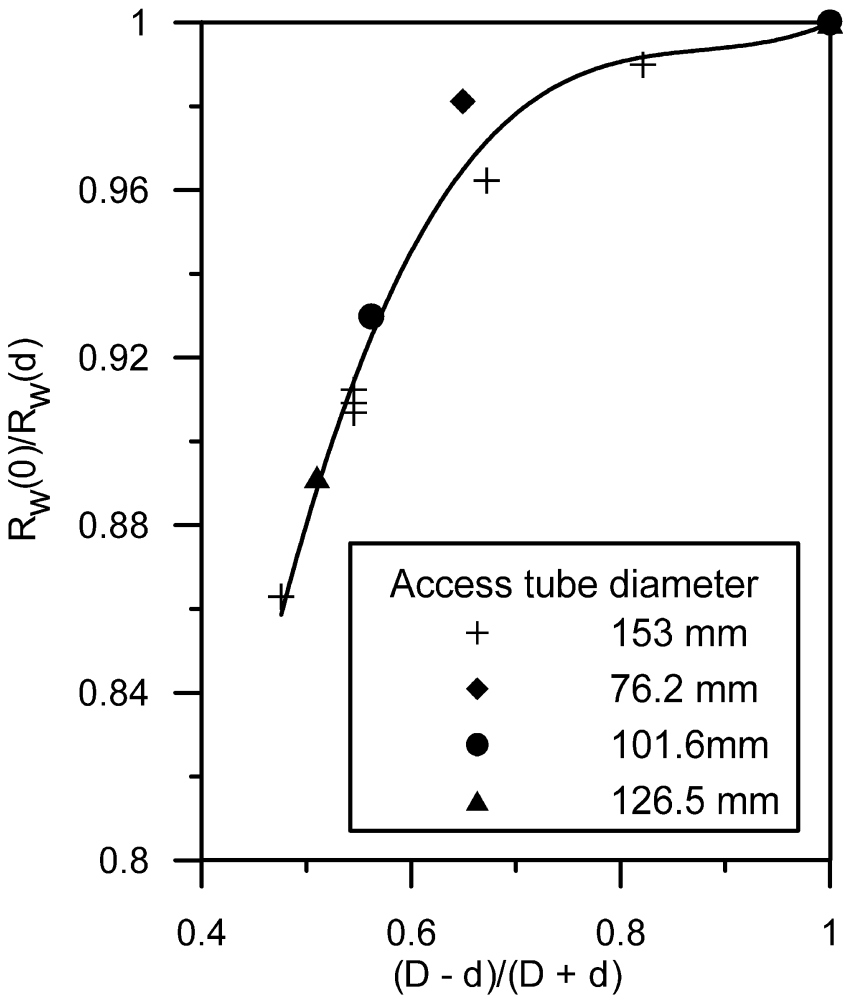

Fig. 4. The effect of offsetting the source/detector unit in the access tube.

have been replotted against the scale ratio $\rho_{\mathrm{s}} / D \rho_{\mathrm{w}}$ and fit well onto the quadratic curve, despite the fact that a centring device was not used. The curve possibly deviates from the data for very low scale ratios. A power law $\left(R_{D}-42\right)=42.2(D)^{-x}\left(\rho_{\mathrm{s}} / \rho_{\mathrm{w}}\right)^{x}$ fits the ice and water data with $r^{2}=0.99$ and passes through the air-count point, but at higher scale ratios the count rate is significantly overestimated. The best-fit value for the ice and water data is $x=$ $0.98 \pm 0.04$. If the same power law (using an air count of 42 ) is fitted to the D98 core data alone, the best-fit value is $x=1.30 \pm 0.04$ with $r^{2}=0.90$. This is consistent with the value of 1.28 for the Nuclear-Chicago Probe (Anderson and others, 1962; Leighty, 1965) based on a similar range of gravimetric snow densities with similar experimental error. Taking all the data together, the best value of the exponent is $x=1.11 \pm 0.03$ with $r^{2}=0.93$. However, the power-law form is in fact not the best choice for an empirical calibration equation. The quadratic form is more accurate over a larger range of scale ratio.

We have also conducted a series of experiments to determine the effect of offsetting the source/detector unit in the access tube. The proportional offset is defined as $(D-d) /$ $(D+d)$, where $d$ is the distance of the centre of the source/ detector unit from the axis of the tube. The normalized water count at offset $d$ is $R_{\mathrm{W} D}(d) / R_{\mathrm{W} D}(0)$. Figure 4 shows a quadratic curve, fitted to all the experimental data except one anomalous point, with coefficients $a_{0}=-0.076, a_{1}=$ $3.45, a_{2}=-3.77$ and $r^{2}=0.995$. Offsetting the source increases the count rate, quite significantly in the case of the larger-diameter tubes. From these data it is clear that the position of the source must be known for accurate measurements of density in ice-core boreholes. The source must be either centred using a centring device or held firmly against the wall of the borehole.

In summary, in this section we have shown that the 


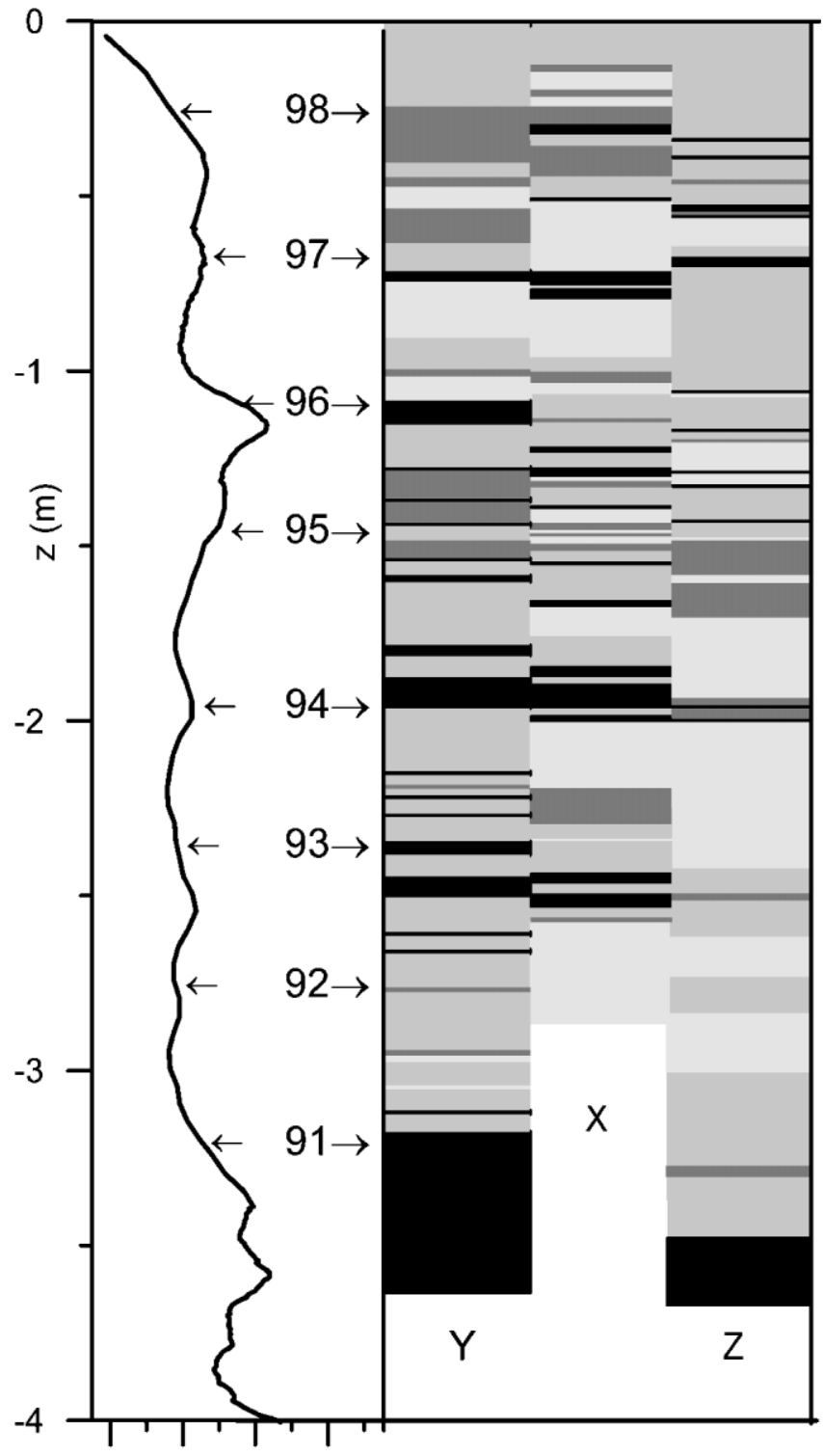

\section{$\begin{array}{llll}0.2 & 0.4 & 0.6 & 0.8\end{array}$}

$\rho_{\mathrm{s}} / \rho_{\mathrm{w}}$

Fig. 5. A density profile measured with the neutron probe with nearby stratigraphic records. Light-grey bands represent depth hoar; dark grey bands represent icy layers ("ice-firn"). Refrozen melt layers are shown as black bands. All other snow types are represented by mid-grey bands. The depth of the summer surface for the years 1991-98 determined independently by R. M. Koerner is also shown.

Wallingford neutron probe can be used to measure snow density on the $10 \mathrm{~cm}$ scale and have produced an accurate calibration curve for the probe which allows the effect of the diameter of the access tube to be taken into account.

\section{MEASUREMENTS OF SNOW STRATIGRAPHY}

In the past it has been assumed that, as in the case of soil moisture measurements, centimetre-scale variations in snow density cannot be resolved. This is because neutron scattering occurs throughout a sphere surrounding the source, of about $15-30 \mathrm{~cm}$ radius, depending on density or water content. The flux of slow neutrons at the detector is a weighted function of density over the sphere. For soil moisture measurements with the Wallingford probe it has become standard practice to assume that the optimum spacing of readings is $10-15 \mathrm{~cm}$ and no greater resolution can be obtained by decreasing this spacing (Bell, 1987). Probably for this reason, in the past snow-density measurements with the Wallingford and other neutron probes have been made at similar, or greater, intervals (e.g. Anderson and others, 1962; Leighty, 1965; Kane, 1969). However, we have found that provided that the amplitude of the density change is sufficiently large, centimetre-scale variations can be distinguished. This opens up the possibility for using the probe to determine detailed snow-cover stratigraphy.

To demonstrate the potential of the probe for stratigraphic measurement, we present two examples of profiles taken on Devon Ice Cap as part of a collaborative study of mass-balance and surface-density fluctuations with the Geological Survey of Canada's National Glaciology Programme.

The first example shows snow density, measured using the Wallingford probe using $5 \mathrm{~cm}$ diameter access holes drilled to $10 \mathrm{~m}$ depth using a Kovacs auger. The count rate was measured over a $64 \mathrm{~s}$ period at vertical spacings of $1 \mathrm{~cm}$ down each hole. The density has been calculated using the empirical calibration equation for uncontaminated snow given in the previous section. (The effect of natural impurities, especially dust containing boron which has a large absorption cross-section for thermal neutrons, has been calculated and found to be negligible.) Figure 5 shows the upper part of a profile $(\mathrm{Cl})$ with three stratigraphic records (X,Yand Z). The profile $\mathrm{Cl}$ was measured first and then two snow pits dug on either side of the augered access hole and faced back to $30 \mathrm{~cm}$ from the hole. Record X comes from the south wall of the north snow pit, and records $\mathrm{Y}$ and $\mathrm{Z}$ from the north wall of the south snow pit. $\mathrm{X}$ and Yare separated by $65 \mathrm{~cm}$; $Y$ and $Z$ by $175 \mathrm{~cm}$. The difference between the stratigraphic records is quite marked, although it is possible that in some cases the snow has been wrongly classified (e.g. at $-1 \mathrm{~m}$ in record $\mathrm{Z}$ the snow type is probably depth hoar as in $\mathrm{X}$ ). In particular, the ice layers are thicker and more numerous on the west side of the south pit (nearY) than on the east side (near Z). A close examination shows evidence of preferential pathways for vertical flow of meltwater which has subsequently refrozen in horizontal layers of limited extent. This significant small-scale horizontal variability makes it difficult to make an exact comparison of the stratigraphy with the neutron-probe density profile. However, the data suggest that, on the $10-50 \mathrm{~cm}$ scale, troughs of low density in the neutron-probe profile are associated with (autumn) depth hoar, and peaks of high density with (summer) ice-firn, and, on the $1-10 \mathrm{~cm}$ scale, kinks in the profile are associated with thin ice layers.

The year labels mark the position of the summer ice-firn layer determined independently by R. M. Koerner by visual inspection of the snow-pit stratigraphy from Y to Z. It appears that the distance between peaks in the neutron-probe profile can be used to estimate the depth of accumulation for each year. This can be converted to water equivalent since the density is also known. Hence, at sites where there is a sufficiently strong annual variation in snow density, and where any summer melt remains within the annual accumulation layer, the neutron probe has the potential to determine an annual mass-balance series.

The second example concerns a "proof-of-concept" experiment undertaken in 2001. A Wallingford source/detector system was incorporated in a geophysical logging system 
$\delta^{18} \mathrm{O}$

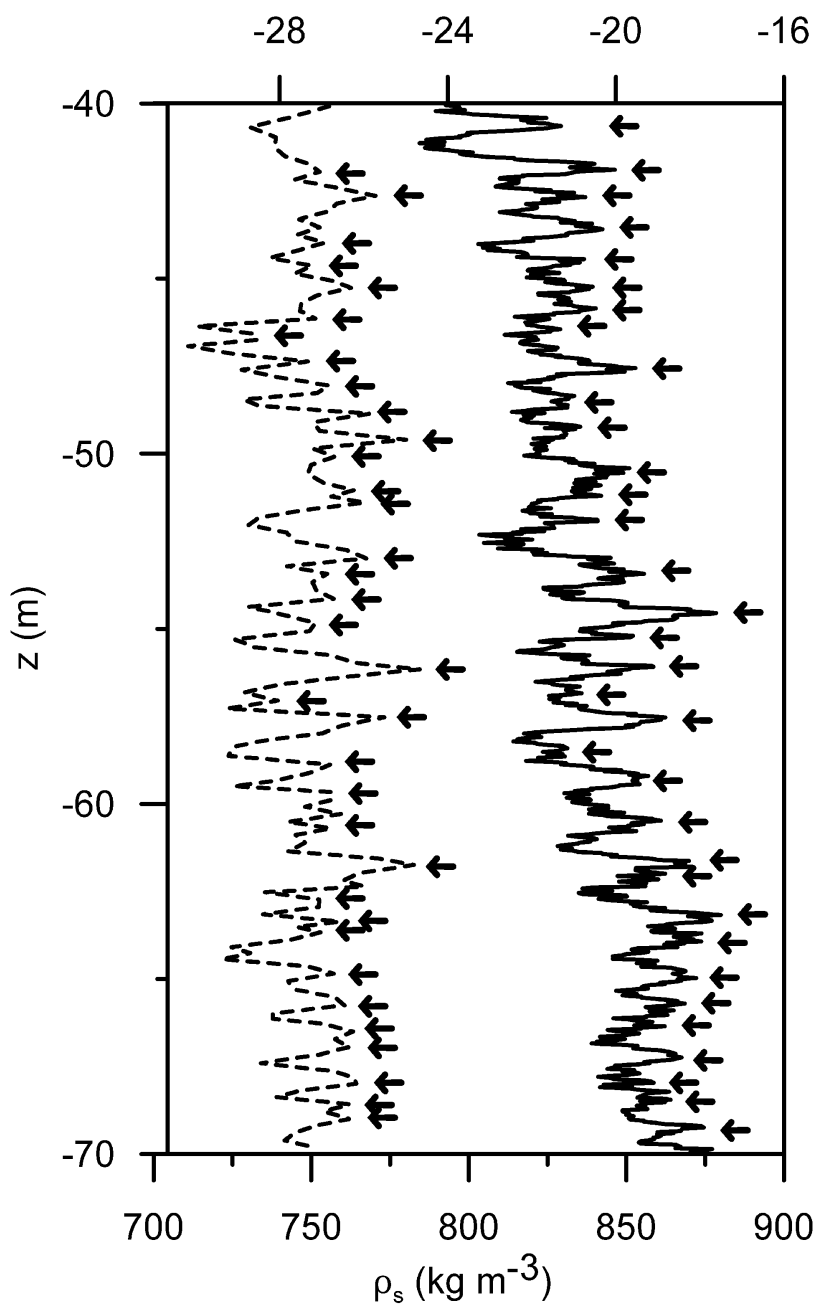

Fig. 6. The variation of $\delta^{18} \mathrm{O}$ in a core taken on Devon Ice Cap (dashed line) and the variation in density measured by profiling the borehole (solid line). Summer peaks are marked with an arrow.

developed by "GeoVista". Figure 6 shows density derived from neutron-probe profiling down a $9 \mathrm{~cm}$ diameter borehole drilled in 1998 and the oxygen-18 isotopic record from a core taken nearby. At this depth the ice is some 300 years old. The present-day accumulation at the site is $20 \mathrm{~cm}$ w.e. $\mathrm{a}^{-1}$, which translates to a depth of $24 \mathrm{~cm}$ of firn of density $850 \mathrm{~kg} \mathrm{~m}^{-3}$. The variation in isotopic concentration in Figure 6 does not resolve all the summer peaks, partly because of the diffusion to be expected at this depth (e.g. Hammer, 1989) and partly because the resolution of the samples is too low. Similarly the density record does not show a high density peak for every summer. However, when the depths of the peaks marked on each curve are compared, a good correspondence is obtained $\left(r^{2}=0.99\right.$ for a straight line plotted through 33 points). This suggests that both records are affected by amount of summer melt, high melt years producing strong peaks in $\delta^{18} \mathrm{O}$ and in density. The implication is that in this case counting density peaks is as accurate a method of deriving the depth-time relationship (and hence the accumulation series) as counting isotope peaks. At this site both isotope and density peaks can be identified down to $170 \mathrm{~m}$ depth. A detailed comparison of isotope and density data over the whole depth range will be presented elsewhere.

\section{FUTURE DEVELOPMENT OF THE SYSTEM}

In order to correct for missed peaks, reference horizons (e.g. radioactive debris, volcanic acids) must be identified. One well-established way of identifying volcanic layers is by electrical profiling. This can be done on the core or within the borehole. Down-borehole gamma probes can also be used to detect dust layers. Thus, for best results, the neutron probe should be part of a geophysical logging system which allows several sondes to be used together in one pass.

The laboratory experiments have shown that knowledge of the diameter of the access hole and the position of the neutron probe within it is vital for accurate density measurement. When small-diameter access tubes are used (as in the measurement of soil water content) down-hole variation may be limited. However, holes left after ice-core drilling can vary in diameter by up to $10 \mathrm{~mm}$ (see, e.g., Tchistiakov and others, 1994). We therefore conclude that the neutronprobe source/detector unit should be combined with a borehole-measuring caliper. Such calipers can measure diameter to better than $0.4 \mathrm{~mm}$ (which would allow density to be determined to $\pm 10 \mathrm{~kg} \mathrm{~m}^{-3}$ rather than the $\pm 50 \mathrm{~kg} \mathrm{~m}^{-3}$ of the gravimetric method). Furthermore, the source/detector unit must either be housed within a centring device or held against one wall of the borehole so that it is always at maximum offset.

We have shown it is possible to observe layering on the 1$10 \mathrm{~cm}$ scale provided that a counting period of at least $64 \mathrm{~s}$ is used. An automatic, continuous profiling system working at a speed of $\approx 2 \mathrm{~cm} \mathrm{~min}^{-1}$ is therefore needed. (This is a factor of 200 times slower than the speed used by Kane (1969) to determine depth-density relations in Antarctica where the annual variation of snow density was not resolved.) Because the time taken to profile a deep borehole will be considerable, all the measurements must be taken in one pass. Development of software to deconvolute the count-rate signal will increase the resolution of the density data.

\section{GONGLUSIONS}

Measurement of the variation of snow and ice density with depth is required to obtain time series of accumulation. The standard gravimetric technique, using samples cut from an ice core, is slow and inaccurate at low densities. A new instrument for rapid, automatic and non-destructive density profiling in the field has now been developed. It can be deployed in rapidly augered access holes, making it feasible to collect the volume of data required to define the spatial and temporal variability of density in natural snow covers. Furthermore, in areas with strong annual variation in density, the instrument can be used to determine accumulation series directly without the necessity of chemical or isotopic analysis of core samples.

The neutron probe can also support other modern methods of mass-balance measurement by providing good density profiles to connect surface elevation to mass-balance change, to interpret radar reflection surfaces and to validate altimeter data.

\section{ACKNOWLEDGEMENTS}

Aspects of this work have been supported by the U.K. Natural Environment Research Council (Connect A grant GR9/CO127 to E.M.M. and J.D.C.) and The Leverhulme 
Trust (grant to E.M.M.). Logistics support was provided by the Canadian Polar Commission/Polar Continental Shelf Project (PCSP) Arctic-Antarctic exchange scheme. This paper is PCSP publication No. 03802. We are grateful to scientists of the Geological Survey of Canada National Glaciology Programme for making core data available, to R. F. Lyman for assistance in the field and to J. Schwander and another referee for helpful comments. D. A. Peel was the Scientific Editor.

\section{REFERENCES}

Anderson, H.W., P. M. McDonald and L. W. Gay. 1962. Gamma and neutron probes for measuring hydrologic characteristics of snow. Am. Geophys. Union Trans., $\mathbf{4 3}(4), 450$.

Bell, J. P. 1969. A new design principle for neutron soil moisture gauges: the Wallingford Neutron Probe. Soil Sci., 103(3), 160-164.

Bell, J. P. 1987. Neutron probe practice. Institute of Hydrology Report 19. Wallingford, Institute of Hydrology.

Gerland, S., H. Oerter, J. Kipfstuhl, F. Wilhelms, H. Miller and W. D. Miners. 1999. Density log of a $181 \mathrm{~m}$ long core from Berkner Island, Antarctica. Ann. Glaciol., 29, 215-219.

Hammer, C. U. 1989. Dating by physical and chemical seasonal variations and reference horizons. In Oeschger, H. and C. C. Langway, Jr, eds. The environmental record in glaciers and ice sheets. Chichester, etc., John Wiley and Sons, 99-121.

Institute of Hydrology. 1981. User's handbook for the Institute of Hydrology Neutron Probe System. Institute of Hydrology Report 79. Wallingford, Institute of Hydrology.

Kane, H. S. 1969. A neutron probe for the determination of snow density and its use in Antarctica. Institute of Polar Studies Report 28, Columbus, $\mathrm{OH}$, The Ohio State University.

Koerner, R. M. 1997. Some comments on climatic reconstructions from ice cores drilled in areas of high melt. f. Glaciol. 43 (143) 90-97.

Leighty, R. D. 1965. Nuclear measurement of snow density. CRREL Spec. Rep. 74.

Ølgaard, P. L. 1965. On the theory of the neutronic method for measuring the water content in soil. Risö Report 97. Roskild, Danish Atomic Energy Commission.

Tchistiakov, V. K., A. Kracilev, V. Ya. Lipenkov, J. Ph. Balestrieri, C. Rado and J. R. Petit. 1994. Behaviour of a deep hole drilled in ice at Vostok Station. In Watanabe, O., ed. Ice drilling technology. Proceedings of the Fourth International Conference on Ice Drilling Technology, Tokyo, Japan, April 20-23, 1993. Tokyo, National Institute of Polar Research, 247-255.

Zagarodnov, V., J. J. Kelley, L. Thompson and O. Watanabe. 1994. Continuous study of an ice core. In Watanabe, O., ed. Ice drilling technology. Proceedings of the Fourth International Conference on Ice Drilling Technology, Tokyo, Japan, April 20-23, 1993. National Institute of Polar Research, Tokyo, 281-289.

MS received 6 December 2002 and accepted in revised form 9 September 2003 